\title{
A SINS/DVL/USBL Integrated Navigation and Positioning loT System with Multiple Sources Fusion and Federated Kalman Filter
}

Qinghua Luo (D luoqinghua081519@163.com)

Harbin Institute of Technology Weihai Campus: Harbin Institute of Technology Weihai https://orcid.org/0000-0002-0168-1245

\section{Xiaozhen Yan}

Harbin Institute of Technology Weihai Campus: Harbin Institute of Technology Weihai

Chenxu Wang

Harbin Institute of Technology Weihai Campus: Harbin Institute of Technology Weihai

\section{Yang Shao}

Harbin Institute of Technology Weihai Campus: Harbin Institute of Technology Weihai

\section{Zhiquan Zhou}

Harbin Institute of Technology Weihai Campus: Harbin Institute of Technology Weihai

Jianfeng Li

Harbin Institute of Technology Weihai Campus: Harbin Institute of Technology Weihai

\section{Cong Hu}

Guilin University of Electronic Technology

\section{Chuntao Wang}

Shandong New Beiyang Information Technology Co. Ltd

Jinfeng Ding

Shandong New Beiyang Information Technology Co. Ltd.

\section{Research Article}

Keywords: Underwater positioning, Federated Kalman filters, Integrated Positioning

Posted Date: December 3rd, 2021

DOI: https://doi.org/10.21203/rs.3.rs-1111330/v1

License: (c) (1) This work is licensed under a Creative Commons Attribution 4.0 International License.

Read Full License 


\title{
A SINS/DVL/USBL Integrated Navigation and Positioning IoT System with Multiple Sources Fusion and Federated Kalman Filter
}

\author{
Qinghua Luo*, Xiaozhen Yan, Chenxu Wang, \\ Yang Shao, Zhiquan Zhou, Jianfeng Li \\ School of information science and engineering \\ Shandong Institute of shipping, Harbin Institute of \\ Technology, Weihai \\ Weihai, China \\ *Corresponding author: luoqinghua081519@163.com
}

\author{
Cong $\mathrm{Hu}$ \\ Guangxi Key Laboratory of Automatic Detecting \\ Technology and Instruments \\ Guilin University of Electronic \\ Guilin, China \\ yiqi@guet.edu.cn
}

\author{
Chuntao Wang, Jinfeng Ding \\ Shandong New Beiyang Information Technology Co., Ltd. \\ Weihai, China \\ wangchuntao@newbeiyagn.com, dingjinfeng@ newbeiyagn.com
}

\begin{abstract}
The navigation and positioning subsystem offers important position information for an autonomous underwater vehicle (AUV) system. It plays a crucial role during the underwater exploration and operations of AUV. Many scholars research underwater navigation and positioning. And many promising methods and systems were presented. However, as the diversity of ocean environment, the random drift of the gyroscope, error accumulation, the diversity of tasks, and other negative factors, the navigation and positioning result is uncertain and incredible. The accuracy, stability and robustness are not guaranteed, which can not meet the increasing application requirement. Therefore, we put forward a SINS/DVL/USBL integrated navigation and positioning IoT system with multiple resource fusion and a federated Kalman filter. In this method, we first present an improved SINS/DVL combined subsystem with filtering gain compensation strategy. The accuracy and stability of the navigation and position system can be enhanced. Secondly, We proposed a USBL positioning subsystem with the Kalman filtering acoustic signals to improve USBL positioning performance. Lastly, we present a federated Kalman Filter to fuse the positioning information from the SINS/DVL combined positioning subsystem and the USBL positioning subsystem. Through the above three methods, we can improve the positioning accuracy and robustness. Comprehensive simulation results indicated the feasibility and effectiveness of the proposed SINS/DVL/USBL integrated navigation and positioning system.
\end{abstract}

Keywords-Underwater positioning; Federated Kalman filters; Integrated Positioning

\section{INTRODUCTION}

In marine-related research fields, autonomous underwater vehicle (AUV) is widely applied to both civil and military-strategic areas [1-2]. And navigation or positioning information is the key for the AUV to find out where it is and where to go [2-4]. The navigation and positioning system determines the efficiency and accuracy when AUV performs underwater missions [5]. However, as the diversity of the ocean environment and the AUV's limitation, it is hard to realize the high-precision navigation and positioning for AUV. And this can not satisfy the increasing needs from applications [5-6]. Since there are many different kinds of navigation and positioning subsystems that have specific characteristics respectively [7], such as SINS, DVL, visual navigation, and USBL [8], we can integrate these subsystems to enhance the overall accuracy and the robustness of the positioning method. Through an integrated approach, we can get higher accuracy and stability of the navigation and positioning system in a long time by performing information fusion and filter correction.

The amount of computation in an integrated system increases with the number of navigation and positioning subsystems. The required computation resource maybe not exceptionally high if there are only two navigation and positioning subsystems in an integrated system [9-10]. However, when three or more subsystems integrate into one system, the dimension will increase to a large scale which causes computation resources to rise at an excessively high level [11]. Under such circumstances, an ordinary filter can not meet the requirement. But the federated filter has the advantage of compatibility and decentralized filter processing, which can simplify the computation and increase the stability of navigation and positioning system.

Many scholars have been researching on the combination of multiple navigation subsystems based on the federated filter [12-13]. When the information of three or more navigation subsystems is fused, the dimension of the multi-sensor information fusion method will be very 
high, resulting in the conventional filtering methods not meeting the needs [14]. In that case, the federated filter method simplifies the calculation and improves the system stability through its robust compatibility and dispersion of filters [15].

For these problems, we proposed a SINS/DVL/USBL integrated navigation and positioning system based on a federated filter, which combines an improved SINS/DVL integrated positioning subsystem and an improved USBL positioning subsystem with Kalman filtering phase difference. So we can improve the positioning accuracy and error tolerance ability of the navigation and positioning system. In this paper, we make the following contributions:

(1) We present a SINS/DVL positioning subsystem based on the filtering gain compensation strategy to enhance the navigation accuracy and the dynamic tracking ability.

(2) We propose a phase-difference filtering USBL navigation subsystem to achieve a higher positioning accuracy.

(3) To fuse the proposed improved SINS/DVL subsystem and the USBL subsystem, we present a SINS/DVL/USBL integrated navigation and positioning system with multiple resource fusion and federated Kalman filter.

\section{RELATED WORKS}

With high accuracy and robust requirement from underwater applications and the complex working environment, challenges are present for scholars and researchers on underwater navigation and positioning. They focus on researching the federated filter, which is a multiple-source information fusion filter developing continuously.

Reference [16] proposed a new federated Kalman adaptive filter. During the information fusion stage, the weighted coefficient was assigned to different states adaptive to enhance the accuracy and robustness. Reference [17] presented a new federated adaptive filter. When there was an outlier during navigation and positioning, factors could be adjusted automatically. The algorithm could restrain the outlier of navigation and positioning and improve the fault-tolerant ability of the positioning system. Reference [18] presented a transfer alignment method based on a federated filter, which divided the high-dimensional state vector into two parts. This method reduced the dimension of the system state vector and improves the calculation speed. Reference [19] presented an adaptive federated filter based on improved covariance, which derived a real-time estimates of improved covariance according to maximum likelihood estimation criteria. And it introduced a scaling factor in each local filter to enhance the adaptive capability of the whole system. Reference [20] took a SINS/GPS/CNS/Radar integrated system as an example. It calculated the parameters corresponding to each state with a dual-state detection. Reference [21] introduced a joint filter to fuse data. When the speed-accuracy of DVL decreased. And the CNS could not work under severe weather conditions. The INS/CNS/DVL combined system could operate stably. Reference [22] designed a federated unscented Kalman filter (FUKF) with different vehicle motion models to estimate the vehicle attitude. The result is promising. Reference [23] designed a federated hybrid filter. In the filter, a minimum variance criterion is utilized to fuse the estimate of each local filter. Reference [24] presented a federated filter for multiple-sensor crosscorrelations strategy. An adaptive filter was utilize as a local filter, which can conquer the performance degradation .To enhance the accuracy and robustness, reference [25] proposed a federated filter with a feedback scheme for a GNSS/INS/visual odometry combined positioning system. In reference [26], a federated Kalman filter (FKF) was applied to indoor positioning. the FKF estimate the target's location. And the distance is estimated through the received signal strength (RSS), which may lead to large positioning error.

For the problem of low accuracy and poor fault tolerance under complex tasks, we can utilize a federated filter based on multiple source information fusion to optimize the system and improve the system performance. Based on the above analysis, we study the information fusion technology of underwater vehicles in a complicated task environment. To achieve higher positioning accuracy, stability and robustness, we put forward a SINS/DVL/USBL integrated navigation and positioning system with federated Kalman filter.

\section{THE FEDERATED KaLMAN FILTER}

To gain relatively high positioning accuracy, we take full advantage of the SINS, DVL, and USBL for navigation and positioning information fusion. As shown in Fig.1, the framework of the proposed integrated navigation and positioning system is composed of three subsystems: an Integrated SINS/DVL subsystem, an improved USBL subsystem, and a global combined federated Kalman filter. The navigation and positioning output is also fed back to the Improved SINS/DVL integrated subsystem for correction and compensation. So we can enhance the positioning accuracy and robustness. 


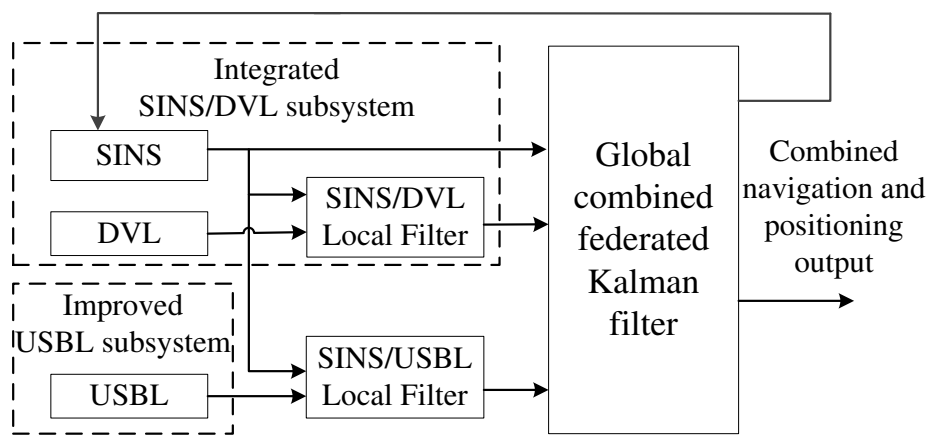

Fig. 1 The framework of the combined navigation and positioning system

Integrated SINS/DVL: We integrated a SINS and a DVL navigation and positioning model with a SINS/DVL local filter. In this local filter, we make full use of a filter gain compensation-based Kalman adaptive filter for rapid changing of UUV working status. In this case, we can obtain high positioning accuracy and stability. The SINS part serves as a critical reference for the SINS/DVL local filter and the SINS/USBL local filter as well.

Improved USBL: Due to environmental noise and multi-path propagation of sonar signal, the phase difference information is affected by different degrees of noise, which leads to low positioning accuracy. In the subsystem, considering the error sources and its affecting mechanism, we present a USBL positioning subsystem through the phase difference and Kalman filtering to enhance positioning performance.

Global combined federated Kalman filter: To fuse the SINS/DVL and USBL positioning subsystem, we utilize a federated Kalman filter, which performs as a multi-source fusion center of the positioning information. We can get the global best estimation of navigation and positioning. The global positioning results are also fed backs to the SINS subsystem to correct positioning error.

\section{A. SINS/DVL Combined Navigation and Positioning Subsystem}

In this subsystem, we utilize an indirect filter to perform information fusion. In the indirect filter, we treat the deviation between the SINS output and the DVL output as a reference value. We provide the error estimation result of combined navigation to SINS for correcting. We call this output correction. Also, the error estimation is feedback filter for system correction. We call it feedback correction. We combine the output correction and the feedback correction. We show the framework of the proposed improved SINS/DVL navigation and positioning subsystem in Fig. 2.

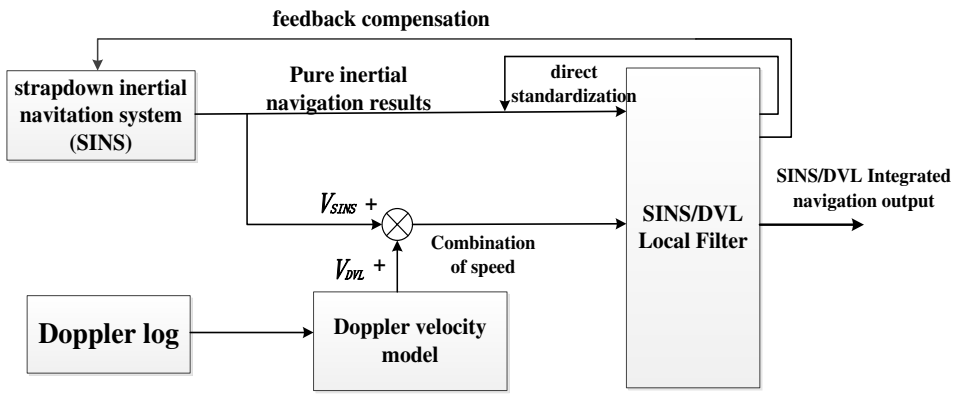

Fig. 2 The framework of the integrated SINS/DVL subsystem

As showed in Fig.2, the framework of the integrated SINS/DVL positioning subsystem comprises a SINS, a DVL, and a SINS/DVL filter. To improve the accuracy and stability of the integrated navigation and positioning subsystem, we present a filter gain compensation adaptive filter to perform information fusion. In this section, we first model the SINS/DVL integrated navigation and positioning through error analysis. Then we present a filtering gain compensation-based adaptive filter to handle the navigation and positioning parameters. And we can achieve accurate positioning error information. Finally, with the error information, through resolving, we obtain accurate navigation and positioning result.

(1) SINS/DVL integrated positioning modelling

The error model of the combined navigation and positioning subsystem comprises the SINS error model and DVL error model. We select the positioning error, the velocity error, the drift angle error, the velocity offset error, the scale factor error as the state variables to the filer. In 
addition, the DVL provides the velocity and drift angle of the AUV.

We denote the system state variable as

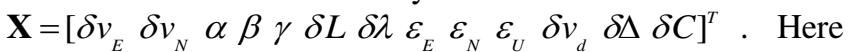
$\delta v_{E}$ and $\delta v_{N}$ denote the speed error in the east and the north. $\alpha 、 \beta 、 \gamma$ represent the AUV misalignment angle, $\delta L$ and $\delta \lambda$ denote latitude and longitude error, $\varepsilon_{E} 、 \varepsilon_{N}$ 、 $\varepsilon_{U}$ are gyroscope drift in the east, the north and the up direction. $\delta v_{d}$ is measuring velocity offset error for Doppler, $\delta \Delta$ is bias angle error, $\delta C$ is the scale factor error.

We define the east speed error $\delta v_{E}$ and north speed error $\delta v_{N}$ :

$$
\begin{gathered}
\delta v_{E}=v_{N} \tan L \cdot \delta v_{E} / R_{\mathrm{N}}+\left(2 \Omega \sin L+v_{E} \tan L / R_{\mathrm{N}}\right) \delta v_{N} \\
+\left(2 \Omega \cos L v_{N}+v_{N} v_{E} \sec ^{2} L / R_{\mathrm{N}}\right) \delta L-\beta g+\Delta a_{E} \\
\delta v_{N}=\left(2 \Omega \sin L+v_{E} \tan L / R_{\mathrm{N}}\right) \delta v_{E} \\
+\left(2 \Omega \cos L v_{E}+v_{E}^{2} \cdot \sec ^{2} L / R_{\mathrm{N}}\right) \delta L+\alpha g+\Delta a_{N}
\end{gathered}
$$

We calculate the platform misalignment angle in (3-5): $\alpha=-\delta v_{N} / R-\gamma\left(\Omega \cos L+v_{E} / R\right)+\beta\left(\Omega \sin L+v_{E} \tan L / R\right)+\varepsilon_{E}$

$$
\beta=-\alpha\left(\Omega \sin L+v_{E} \tan L / R\right)-\delta L \Omega \sin L+\delta v_{E} / R
$$$$
-\gamma v_{E} / R+\varepsilon_{N}
$$

$\gamma=\delta L\left(\Omega \cos L+v_{E} \sec ^{2} L / R\right)+\delta v_{E} \tan L / R+\beta v_{N} / R$ $+\alpha\left(\Omega \cos L+v_{E} / R\right)+\varepsilon_{U}$

We define the position error in (6):

$$
\begin{gathered}
\delta L=\delta v_{N} / R \\
\delta \lambda=\delta v_{E} \sec L / R+v_{E} \tan L \sec L \delta L / R
\end{gathered}
$$

We calculate the gyro drift in the east, in the north and in the up direction as follows:

$$
\begin{gathered}
\varepsilon_{E}=-\beta_{E} \varepsilon_{E}+w_{E} \\
\varepsilon_{N}=-\beta_{N} \varepsilon_{N}+w_{N} \\
\varepsilon_{U}=-\beta_{U} \varepsilon_{U}+w_{U}
\end{gathered}
$$

Here $\beta_{E}^{-1}, \beta_{N}^{-1}, \beta_{U}^{-1}$ is error related time of the gyroscope in the east, the north, and the up direction.

We define the Speed error $\delta v_{d}$, the drift angle error $\delta \Delta$ and the scale error $\delta C$ of the Doppler log as follows:

$$
\begin{gathered}
\delta v_{d}=-\beta_{d} \delta v_{d}+w_{d} \\
\delta \Delta=-\beta_{\Delta} \delta \Delta+w_{\Delta} \\
\delta C=0
\end{gathered}
$$

We describe the state equation in (14):

$$
\mathbf{X}_{S I N S / D V L}=\mathbf{F}_{S I N S / D V L} \mathbf{X}_{S I N S / D V L}+\mathbf{G}_{S I N S / D V L} \mathbf{W}_{S I N S / D V L}
$$

)

We define the state equation of SINS in (15):

$$
\mathbf{W}_{\text {SINS }}=\left[\begin{array}{lllllllllllll}
0 & 0 & a_{E} & a_{N} & 0 & 0 & 0 & w_{E} & w_{N} & w_{U} & w_{d} & w_{\Delta} & 0
\end{array}\right]^{T}
$$

In (14), we calculate the transfer matrix as follow:.

$$
\begin{aligned}
\mathrm{F}_{S I N S / D V L} & =\left[\begin{array}{l:ll}
\mathbf{F}_{7 \times 7} & \mathbf{0}_{4 \times 3} & \mathbf{0}_{4 \times 3} \\
\hdashline \mathbf{I}_{3 \times 3} & \mathbf{0}_{3 \times 3} \\
\hdashline \mathbf{0}_{6 \times 7} & \mathbf{F}_{6 \times 6}
\end{array}\right] \\
\mathbf{F}_{7 \times 7}= & {\left[\begin{array}{lllllll}
0 & 0 & 0 & F_{14} & 0 & 0 & 0 \\
F_{21} & 0 & F_{23} & 0 & 0 & 0 & 0 \\
F_{31} & 0 & F_{33} & F_{34} & 0 & -g & 0 \\
F_{41} & 0 & F_{43} & 0 & g & 0 & 0 \\
0 & 0 & 0 & F_{54} & 0 & F_{56} & F_{57} \\
F_{61} & 0 & F_{63} & 0 & F_{65} & 0 & F_{67} \\
F_{71} & 0 & F_{73} & 0 & F_{75} & F_{76} & 0
\end{array}\right] }
\end{aligned}
$$

The difference between the velocity error of SINS and DVL is taken as the observation of the measurement equation which is defined as follow:

$$
\begin{aligned}
\mathbf{Z}_{S I N S / D V L} & =\left[\begin{array}{l}
\delta v_{E}-\delta v_{d E} \\
\delta v_{N}-\delta v_{d N}
\end{array}\right] \\
& =\mathbf{H}_{S I N S / D V L} \mathbf{X}_{\text {SINS/DVL }}+\mathbf{V}_{\text {SINS/DVL }}
\end{aligned}
$$

We define the transfer matrix in (20):

$$
\mathbf{H}_{S I N S / D V L}=\left[\begin{array}{lllllllllllll}
0 & 0 & 1 & 0 & 0 & 0 & -v_{N} & 0 & 0 & 0 & -\sin K_{d} & -v_{N} & -v_{E} \\
0 & 0 & 0 & 1 & 0 & 0 & v_{E} & 0 & 0 & 0 & -\cos K_{d} & v_{E} & -v_{N}
\end{array}\right]
$$

$$
\mathbf{V}_{S I N S / D V L}=\left[\begin{array}{ll}
v_{E} & v_{N}
\end{array}\right]^{T}
$$

We define the variance matrix of noise:

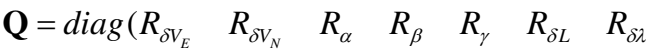

$$
\begin{aligned}
& \left.\begin{array}{llllll}
R_{\varepsilon E} & R_{\varepsilon N} & R_{\varepsilon U} & R_{\delta V d} & R_{\delta \triangle} & R_{\delta C}
\end{array}\right)
\end{aligned}
$$

We calculate the variance matrix of measurement noise:

$$
\mathbf{R}=\operatorname{diag}\left(\begin{array}{ll}
R_{V_{N}} & R_{V_{E}}
\end{array}\right)
$$

(2) Filtering Gain Compensation

The gain $\mathbf{K}$ determines the correction value of state estimation on each moment, which is positively correlated with the correction value. As the system state changes with time constantly. The estimation value of the state can not track the state changing in time, which will lead to a significant positioning error, especially when the state changes rapidly. So we combine the gain compensation and adaptive Kalman filter. And we present a gain compensation based adaptive Kalman filtering method, where the gain compensation and adaptive Kalman filter are combined.

We denote the filter gain as $\mathbf{K}_{n}$. At the time $k$, we compensate the original filtering gain with $\mathbf{K}_{g}$ :

$$
\mathbf{K}_{n}(k)=\mathbf{K}(k)+\mathbf{K}_{g}(k)
$$

We update the filter equation in (25):

$$
\hat{\mathbf{K}}(k+1)=\hat{\mathbf{K}}(k+1 / k)+\hat{\mathbf{K}}(k+1)[\hat{\mathbf{Z}}(k+1)-\hat{\mathbf{Z}}(k+1 / k)](25)
$$

For the stability and robustness of the positioning system, the filtering gain $\mathbf{K}_{n}$ should increase to track the rapid changing of motion and the system state. When the 
state is stable, the filtering gain can decrease to $\mathbf{K}_{g}$.We also define the $\mathbf{K}_{g}$ in (26):

$$
\mathbf{K}_{g}=\mathbf{K}_{z} \lambda^{\left(k-k_{0}\right)}
$$

Here $\lambda$ is lower than 1 . It determines the increasing scale of the filtering gain. $k_{0}$ is the moment of the state changing rapidly.

(3) SINS/DVL Local Filter

The state equation of the SINS/DVL local filter is defined as follows:

$$
\boldsymbol{X}_{k}^{\&}=\boldsymbol{F}_{k} \boldsymbol{X}_{k}+\boldsymbol{W}_{k}
$$

Where the $\boldsymbol{F}_{k}$ is state transfer matrix, which is determined by the error model of SINS/DVL. $\boldsymbol{W}_{k}$ denotes the system noise.

For the SINS/ DVL integrated positioning subsystem, we take the difference between the calculated velocity of the SINS system and the velocity of DVL as the observation of the measurement equation. And the measurement equation of the SINS/DVL local filter is:

$$
\boldsymbol{Z}_{S I N S / D V L}=\left[\begin{array}{l}
d v_{E}-d v_{d E} \\
d v_{N}-d v_{d N}
\end{array}\right]=\boldsymbol{H}_{S I N S / D V L} \boldsymbol{X}_{S I N S / D V L}+\boldsymbol{V}_{S I N S / D V L}
$$

Where $\boldsymbol{H}_{S I N S / D V L}$ and $\boldsymbol{V}_{S I N S / D V L}$ are defined as follows:

$$
\boldsymbol{H}_{S I N S / D V L}=\left[\begin{array}{lllllllllllll}
0 & 0 & 1 & 0 & 0 & 0 & -v_{N} & 0 & 0 & 0 & -\sin K_{d} & -v_{N} & -v_{E} \\
0 & 0 & 0 & 1 & 0 & 0 & v_{E} & 0 & 0 & 0 & -\cos K_{d} & v_{E} & -v_{N}
\end{array}\right]
$$

$$
\boldsymbol{V}_{\text {SINS/DVL }}=\left[\begin{array}{ll}
v_{E} & v_{N}
\end{array}\right]^{T}
$$

The subsystem adopts the improved adaptive filtering algorithm based on filter gain compensation, where $\boldsymbol{H}_{k}$ is the observation coefficient matrix of the subsystem and $\boldsymbol{V}_{k}$ is the observation noise matrix. Firstly, $\boldsymbol{F}_{k} 、 \boldsymbol{G}_{k} 、 \boldsymbol{W}_{k}$ 、

$\boldsymbol{H}_{k} 、 \boldsymbol{V}_{k}$ and the initial state variable $\boldsymbol{X}$ are substituted into the state equation and measurement equation of the system for one-step prediction, through which the estimation measurement value $\hat{\boldsymbol{Z}}_{k}$ and $\hat{\boldsymbol{X}}_{k, k-1}$ predicted at time $k$ can be obtained. And the error of measurement value $Z_{k}^{o}$ at time $k$ is calculated as follow:

$$
Z_{k}^{o}=\hat{\boldsymbol{Z}}_{k}-\boldsymbol{H}_{k} \hat{\boldsymbol{X}}_{k, k-1}
$$

Then we judge the system is stable or not according to the stable criterion:

$$
\boldsymbol{Z}_{k}^{0} \boldsymbol{Z}_{k}^{6 / 5} \leq \gamma \operatorname{Tr}\left[\boldsymbol{H}_{k} \boldsymbol{P}_{k} \boldsymbol{H}_{k}^{T}+\boldsymbol{R}_{k}\right]
$$

If the criterion (32) holds, the system diverges and gain compensation adaptive filtering should be adopted. Otherwise, the system converges and the gain compensation-based strong tracking Kalman filter should be adopted.

1) Gain compensation based adaptive Kalman filter

By substituting the corresponding parameters and quantities into formulas (33) to (42) for calculation, the steps of the gain compensation-based adaptive filter are as follow:

$$
\begin{gathered}
\hat{\boldsymbol{X}}_{k}=\hat{\boldsymbol{X}}_{k, k-1}+\boldsymbol{K}_{k} \boldsymbol{Z}_{k}^{o} \\
\hat{\boldsymbol{X}}_{k, k-1}=\boldsymbol{F}_{k, k-1} \hat{\boldsymbol{X}}_{k-1}+\hat{\boldsymbol{q}}_{k} \\
\hat{\boldsymbol{Z}}_{k}=\boldsymbol{Z}_{k}-\boldsymbol{H}_{k} \hat{\boldsymbol{X}}_{k, k-1}-\hat{\boldsymbol{r}}_{k} \\
\boldsymbol{K}_{k}=\boldsymbol{P}_{k, k-1} \boldsymbol{H}_{k}^{T}\left(\boldsymbol{H}_{k} \boldsymbol{P}_{k, k-1} \boldsymbol{H}_{k}^{T}+\hat{\boldsymbol{R}}_{k}\right)^{-1} \\
\boldsymbol{P}_{k, k-1}=\boldsymbol{F}_{k, k-1} \boldsymbol{P}_{k-1} \boldsymbol{F}_{k, k-1}^{T}+\hat{\boldsymbol{Q}}_{k-1} \\
\boldsymbol{P}_{k}=\left(\mathbf{I}-\boldsymbol{K}_{k} \boldsymbol{H}_{k}\right) \boldsymbol{P}_{k, k-1}\left(\mathbf{I}-\boldsymbol{K}_{k} \boldsymbol{H}_{k}\right)^{T}+\boldsymbol{K}_{k} \hat{\boldsymbol{R}}_{k} \boldsymbol{K}_{k}
\end{gathered}
$$

Here $\hat{\boldsymbol{X}}_{k}$ is the system state variable. $\hat{\boldsymbol{X}}_{k, k-1}$ denotes the estimation value of the system state variable at the moment $k$ from the $k-1$ moment. $\boldsymbol{F}_{k, k-1}$ represents the state transfer coefficient on $\hat{\boldsymbol{X}}_{k-1} \cdot \boldsymbol{K}_{k}$ is Kalman coefficient of the filtering gain compensation. $\boldsymbol{H}$ denotes the coefficient of the system observation equation. $\boldsymbol{Z}_{k}$ is the observation value at the $k$ moment. $\hat{Z}_{k}$ denotes the estimation value of observation at the $k$ moment. $\boldsymbol{P}_{k, k-1}$ is the covariance of the prior state estimation. $\boldsymbol{P}_{k}$ denotes the covariance of the post-state estimation.

In formulas (33) to (38), $\hat{\boldsymbol{r}}_{k}, \hat{\boldsymbol{R}}_{k}, \hat{\boldsymbol{q}}_{k}$ and $\hat{\boldsymbol{Q}}_{k}$ are calculated from the time-varying noise estimation equation as follows:

$$
\begin{gathered}
\hat{\boldsymbol{r}}_{k}=\left(\mathbf{I}-\boldsymbol{d}_{k-1}\right) \hat{\boldsymbol{r}}_{k-1}+\boldsymbol{d}_{k-1}\left(\boldsymbol{Z}_{k}-\boldsymbol{H}_{k, k-1} \hat{\boldsymbol{X}}_{k, k-1}\right) \\
\hat{\boldsymbol{R}}_{k}=\left(\mathbf{I}-\boldsymbol{d}_{k-1}\right) \hat{\boldsymbol{R}}_{k-1}+\boldsymbol{d}_{k-1}\left(\boldsymbol{Z}_{k}^{o} \boldsymbol{Z}_{k}^{b}-\boldsymbol{H}_{k, k-1} \boldsymbol{P}_{k} \boldsymbol{H}_{k}^{T}\right) \\
\hat{\boldsymbol{q}}_{k}=\left(\mathbf{I}-\boldsymbol{d}_{k-1}\right) \hat{\boldsymbol{R}}_{k-1}+\boldsymbol{d}_{k-1}\left(\hat{\boldsymbol{X}}_{k}-\boldsymbol{F}_{k} \hat{\boldsymbol{X}}_{k-1}\right) \\
\hat{\boldsymbol{Q}}_{k}=\left(\mathbf{I}-\boldsymbol{d}_{k-1}\right) \hat{\boldsymbol{Q}}_{k-1}+\boldsymbol{d}_{k-1}\left(\boldsymbol{K}_{k} \boldsymbol{Z}_{k}^{o} \boldsymbol{Z}_{k}^{b} \boldsymbol{K}_{k}^{T}+\boldsymbol{P}_{k}-\boldsymbol{F}_{k} \boldsymbol{P}_{k-1} \boldsymbol{F}_{k}^{T}\right)
\end{gathered}
$$

2) Gain compensation-based strong tracking Kalman filter

By substituting the corresponding parameters and quantities into formulas (43) to (51) for calculation, the steps of the gain compensation-based strong tracking Kalman filter are as follow:

$$
\begin{gathered}
\boldsymbol{v}_{0(k+1)}=\left\{\begin{array}{cc}
\boldsymbol{Z}_{1} \mathbf{Z}_{1}^{b} & (k=0) \\
\frac{\rho \boldsymbol{v}_{0(k)}+\boldsymbol{Z}_{k+1}^{o} \boldsymbol{Z}_{k+1}^{T}}{1+\rho}(k \geq 1,0 \leq \rho<1)
\end{array}\right. \\
C_{k+1}=\frac{\operatorname{Tr}\left[\boldsymbol{v}_{0(k+1)}-\boldsymbol{R}_{k+1}-\boldsymbol{H}_{k+1} \boldsymbol{Q}_{k} \boldsymbol{H}_{k+1}^{T}\right]}{\sum_{i=1}^{n} \alpha_{i}\left[\boldsymbol{\Phi}_{k+1} \boldsymbol{P}_{k+1} \boldsymbol{\Phi}_{k+1}^{T} \boldsymbol{H}_{k+1}^{T} \boldsymbol{H}_{k+1}\right]} \\
\lambda_{i(k+1)}= \begin{cases}\alpha_{i} C_{k+1}\left(\alpha_{i} C_{k+1}>1\right) \\
1 & \left(\alpha_{i} C_{k+1}<1\right)\end{cases} \\
\lambda_{k+1}=\operatorname{diag}\left[\lambda_{1(k+1)}, \lambda_{2(k+1)}, \ldots, \lambda_{m(k+1)}\right] \\
\boldsymbol{P}_{k, k-1}=\lambda_{k-1} \boldsymbol{\Phi}_{k, k-1} \boldsymbol{P}_{k, k-1} \boldsymbol{\Phi}_{k, k-1}^{T}+\hat{\boldsymbol{Q}}_{k-1} \\
\boldsymbol{K}_{k}=\boldsymbol{P}_{k, k-1} \boldsymbol{H}_{k}^{T}\left(\boldsymbol{H}_{k} \boldsymbol{P}_{k, k-1} \boldsymbol{H}_{k}^{T}+\hat{\boldsymbol{R}}_{k}\right)^{-1}
\end{gathered}
$$




$$
\begin{gathered}
\hat{\boldsymbol{X}}_{k}=\hat{\boldsymbol{X}}_{k, k-1}+\boldsymbol{K}_{k} \boldsymbol{Z}_{k}^{o} \\
\hat{\boldsymbol{X}}_{k, k-1}=\boldsymbol{F}_{k, k-1} \hat{\boldsymbol{X}}_{k-1}+\boldsymbol{G} \boldsymbol{W}_{k, k-1} \\
\boldsymbol{P}_{k}=\left(\mathbf{I}-\boldsymbol{K}_{k} \boldsymbol{H}_{k}\right) \boldsymbol{P}_{k, k-1}\left(\mathbf{I}-\boldsymbol{K}_{k} \boldsymbol{H}_{k}\right)^{T}+\boldsymbol{K}_{k} \hat{\boldsymbol{R}}_{k} \boldsymbol{K}_{k}
\end{gathered}
$$

In this way, the one-step filtering operation is realized through the above adaptive filtering and strong tracking filtering to obtain the estimated value $\hat{X}_{k}$ of the state variable at the next time.

Finally, the federated filter subsystem gains the state variable sequence $\hat{\boldsymbol{X}}=\left\{\hat{\boldsymbol{X}}_{1}, \hat{\boldsymbol{X}}_{2}, \ldots, \hat{\boldsymbol{X}}_{k}, \ldots, \hat{\boldsymbol{X}}_{n}\right\}$, whose final output and output the final result $\hat{\boldsymbol{X}}_{n}$ is the state result after filtering correction at the current time. And the

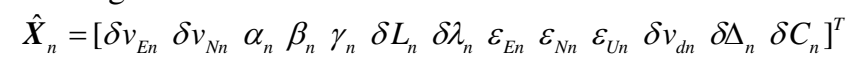
is the correct result of the error. Then we combine the current moment observation value $\boldsymbol{Y}_{n}=\left[\begin{array}{lllll}v_{E n} & v_{N n} & L_{n} & \lambda_{n} & v_{d n}\end{array}\right]^{T}$ of the integrated positioning subsystem. And we achieve the corrected East, North velocity information, longitude and latitude information, and velocity information of DVL.

\section{B. USBL Navigation and Positioning Subsystem}

We improve the USBL positioning method through the phase difference acquisition of the Kalman filter (KF) algorithm. As shown in Figure 3, the system structure mainly consists of three parts: the array element setup part, the signal noise reduction part, and the positioning calculation part.

Firstly, in the array element setup part, we adopt a non-equidistant quaternary [27]. It reuses a specific element three times, improving the efficiency and accuracy of the acoustic signals captured from the underwater target. We utilize the array to obtain the time delay of the received signals from a target object to different elements.

Secondly, in the signal noise reduction part, we adopt a KF algorithm to filter out the white Gaussian noise in the acoustic signals. So we can obtain accurate phase difference information through a phase difference calculation sub-module to provide data for the next step.

Finally, in the positioning calculation part, we obtain the positioning result through the coordinate calculation formula according to the positioning model based on the obtained phase difference between signals with high accuracy.

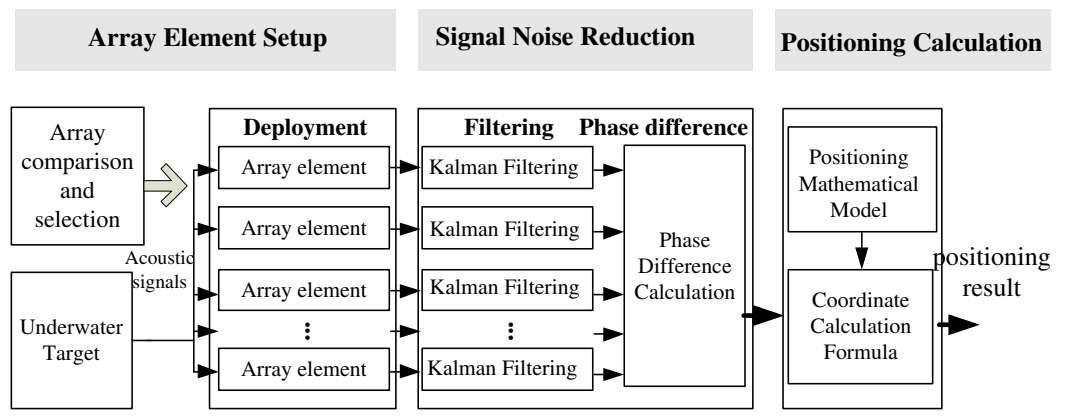

Fig. 3 The framework of the underwater positioning

(1) Array Element Setup

The non-equidistant quaternary array of the USBL system is composed as shown in Figure 4. The distance between array element 1 and element 3 is $d$, which is less or equal to $\lambda / 2$, where $\lambda=80 \mathrm{~mm}$ and the $x$-axis angle $\theta=45^{\circ}$. And the distance between array element 2 and array element 3 is the same as that between array

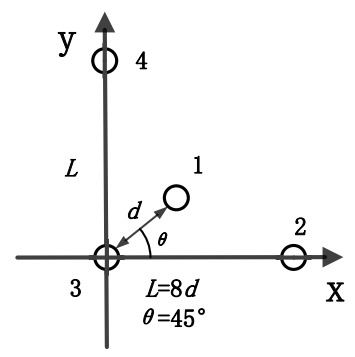

Fig. 4 The USBL positioning system array diagram [27]

element 4 and array element 3, which is $L=8 d$. The advantage of this method is that the acoustic signal received by element 3 is reused three times, which reduces the number of redundant elements, enhances the length of the baseline, and further improves the positioning accuracy of the system. 
We indicate the arrangement of the arrays in Fig 5. Element 3 and 2 are two elements in the $x$-axis, which can receive acoustic signals from the positioning project. As difference propagation distance between two received signals, there is a phase difference between the two received acoustic signals. Considering this case, the specific process of solving the USBL positioning coordinates is as follows:

$$
x_{L}=\left(\lambda \phi_{23} R\right) / 2 \pi L
$$

Where $x_{L}$ is the mapping coordinate of the object in the array. $\phi_{23}$ denotes the phase difference between the arrays on the $x$-axis. $R$ is the distance between the target and the array, which can be obtained directly by the measurement instrument .

In the same calculation manners, we obtain the mapping coordinate $x_{d}$ of element 1 on the $x$-axis through element 3 and element 1 .

$$
x_{d}=\left(\lambda \phi_{13} R\right) / 2 \pi d
$$

Assuming the noise background of each array element in the USBL system is independent, the phase differences $\phi_{i j}$ and $\psi_{i j}$ have the same measurement accuracy $\Delta \phi$, which meets the equation $\Delta \phi_{13}=\Delta \phi_{23}=\Delta \phi$

Equation (53) is the positioning solution formula of the traditional USBL array, and equation (52) is the positioning solution formula of the new USBL array. After differential treatment of equations (1) and (2), we can get:

$$
\begin{aligned}
\Delta x_{d} & =\frac{\lambda R}{2 \pi d} \Delta \phi_{13}=\frac{\lambda R}{2 \pi d} \Delta \phi \\
\Delta x_{L} & =\frac{\lambda R}{2 \pi L} \Delta \phi_{23}=\frac{\lambda R}{2 \pi L} \Delta \phi
\end{aligned}
$$

Formulas (54) and (55) show that choosing an array arrangement with large spacing $L=8 d$ can improve positioning accuracy by eight times. For the new array used in this paper, the problem of multi-value ambiguity of phase difference can be solved by $d$-spacing elements (elements 1 and 3), and positioning accuracy can be solved by $L$-spacing elements (elements 2 and 3 ). The combination of the two methods can ultimately improve the system's accuracy.

\section{(2) Signal Filtering}

Noise will cause a significant interference to the positioning of underwater objects, so it is usually necessary to denoise the obtained underwater acoustic signals to achieve the positioning of underwater targets. To minimize the noise interference and improve the positioning accuracy, the minimum mean square error is selected as the best estimation criterion to reduce the noise received by the sensor. Based on this idea, this paper uses the $\mathrm{KF}$ algorithm to process the obtained signal based on minimum mean square error estimation.
When receiving the signals, we get the sampling sequence $S=\left\{s_{1}, s_{2}, s_{3}, \ldots, s_{k}, \ldots, s_{n}\right\}$ by sampling them. In the above equation, $k$ denotes a serial number, $1 \leq k \leq n$. And the sampling sequence can be normalized as follows:

$$
u_{k}=s_{k} /(\max (S)-\min (S))
$$

In this paper, the KF algorithm is used to filter the sampling sequence $\left\{u_{k}\right\}$ to reduce the noise.

$$
\begin{gathered}
X_{k}=H^{*} X_{k-1}+G^{*} W_{k-1} \\
u_{k}=H^{*} X_{k}
\end{gathered}
$$

Where $k$ is the discrete-time, $X=\left\{X_{1}, X_{2}, X_{3}, \ldots, X_{k}\right.$, $\left.\ldots, X_{n}\right\}$ is the state value of the system and.$X_{k}$ is the state value of the system at time $k . u=\left\{u_{1}, u_{2}, u_{3}, \ldots, u_{k}, \ldots, u_{n}\right\}$ is the observation signal of the system. $W=\left\{W_{1}, W_{2}, W_{3}, \ldots\right.$, $\left.W_{k}, \ldots, W_{n}\right\}$ is the input white noise; and $W_{k}$ is the noise value of the system at time $k, H=1$ is the measurement matrix, and the specific value of the observed value $u$ is the value of the input signal.

KF algorithm mainly consists of two parts: prediction and update. In the prediction part, the filter estimates the state of the current moment based on the state estimates of the previous moment. In the update section, the filter optimizes the predicted values from the prediction section based on the observations of the system state at the current time, and the final more accurate state estimate is obtained. The specific equations of the KF algorithm are as follows:

Time update equation:

$$
\begin{gathered}
\hat{X}_{k}=A \hat{X}_{k-1} \\
P_{k}^{-}=A P_{k-1} A^{T}+Q
\end{gathered}
$$

State update equation:

$$
\begin{gathered}
K_{k}=P_{k}^{-} H^{T}\left(H P_{k}^{-} H^{T}+R\right)^{-1} \\
\hat{X}_{k}=\hat{X}_{k}^{-}+K_{k}\left(u_{k}-H \hat{X}_{k}^{-}\right) \\
P_{k}=\left(I-K_{k} H\right) P_{k}^{-}
\end{gathered}
$$

Where $A$ is the state transition factor of the state variable. The $B$ denotes the input control factor matrix of the control vector. The $H$ represents the system state observation matrix. The $P_{k}^{-}$is the system prior estimate covariance matrix. The $P_{k}$ denotes the system posterior estimate covariance matrix. The $Q$ is the process excitation noise covariance coefficient. The $R$ is the covariance coefficient of process noise. The $I$ denotes the unit matrix. And the $K_{k}$ is the Kalman filter gain factor.

Then we can get the equation as follow:

$$
\hat{u}_{k}=\hat{X}_{k}
$$


Where the $\hat{X}_{k}$ is the estimation value of the state variable. The $\hat{X}_{k}^{-}$is the prior estimation of the state obtained from the state variable.

The KF algorithm described above is used to estimate the state variables of a random process described by a linear random difference equation. The magnitude of the observed value of the system is the input signal value after adding noise. Combining with the established system state equation, the optimal input $\hat{X}_{k}$ value is estimated by re-cursing the variance continuously, and the signal is smoothed.
(3) Positioning Calculation

First, we introduce the traditional phase difference positioning principle. For single-frequency $\mathrm{CW}$ signals, the most common information is based on the phase information obtained when the USBL target is positioned. Then, we calculate the target position through the phase difference of the acoustic signals between the elements. When multiple targets need to be located, different frequencies of acoustic signals can be used to distinguish them. The principle of USBL positioning based on acquiring phase difference of a single-frequency signal is described below in Fig. 5 .

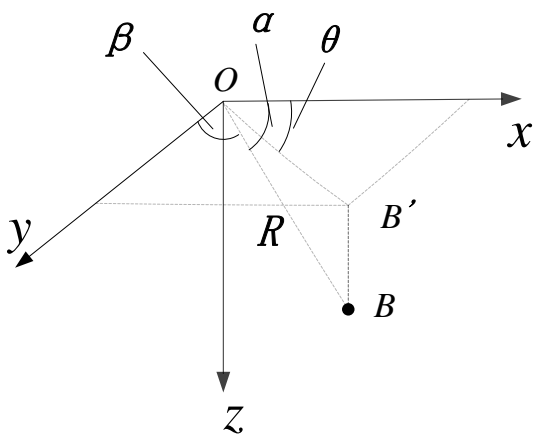

Fig. 5 The principle of positioning

The array elements of the USBL system are placed on the xoy plane, where element 1 is located at the origin of the xoy coordinate system. Element 2 is located on the positive semi-axis of the $x$-axis with a distance of $d$ from the origin of the coordinates. And element 3 is located on the positive semi-axis of the $y$-axis, with a distance of $d$ from the origin of the coordinates. The target is located at $S$ with specific coordinates $(x, y, z)$. After the target emitting an acoustic signal, the array element receives the acoustic signal to locate the target. The system processes the received acoustic signal to get the specific location of the target.

From Figure 5, the $O S$ direction cosine can be expressed as:

$$
\begin{gathered}
R \cos \alpha=x \\
R \cos \beta=y \\
R=\sqrt{x^{2}+y^{2}+z^{2}}
\end{gathered}
$$

Where $\alpha$ is the angle between the $O S$ and the $x$ axis. $\beta$ denotes the angle between the $O S$ and the $y$ axis, and $R$ is the target distance.

In Figure $5, S^{\prime}$ is the projection of the target $S$ on the horizontal plane of xoy. And its angle to the $x$-axis is the horizontal azimuth $\theta$ of the target, which is determined by the formula (68):

$$
\begin{gathered}
\theta=\operatorname{tg}^{-1}(y / x)=\operatorname{tg}^{-1}(\cos \beta / \cos \alpha) \\
r=\sqrt{x^{2}+y^{2}} \\
z=\sqrt{R^{2}-r^{2}}
\end{gathered}
$$

Where $R$ is the target horizontal slant range, and $z$ is the target depth.

Formulas (65) to (70) are the formulas for the positioning solution of USBL. The corresponding parameters of the target object can be calculated according to the above formulas.

Since the USBL array has a very tiny size, we can approximate it by formulas (20) and (21):

$$
\begin{aligned}
& \phi=(2 \pi d \cos \alpha) / \lambda \\
& \Psi=(2 \pi d \cos \beta) / \lambda
\end{aligned}
$$

Where $\lambda$ is the wavelength of the acoustic signal in the water, $d$ denotes the distance between the elements of the array, $\phi$ represents the phase difference between signals received by two adjacent arrays on the $x$ - axis, and $\psi$ is the phase difference of the received signal for two adjacent arrays on the $y$-axis.

By substituting formulas (71) and (72) into formulas (65) and (66), we can obtain the following results:

$$
x=(\lambda \phi R) /(2 \pi d)
$$




$$
y=(\lambda \varphi R) /(2 \pi d)
$$

Where $R$ meets the equation $R=c \times \Delta t / 2$, in which $c$ is the speed of sound propagation in water and $\Delta t$ is the time difference between signal transmission and reception.

\section{Global Combined Federated Kalman Filter}

Suppose the state variable of the federated filter from moment $k-1$ to moment $k$ with state equation:

$$
\boldsymbol{X}_{k}=\boldsymbol{F}_{k, k-1} \boldsymbol{X}_{k-1}+\boldsymbol{W}_{k-1}
$$

The observation equation of the $i$ th subsystem is as follows:

$$
\boldsymbol{Z}_{i k}=\boldsymbol{H}_{i k} \boldsymbol{X}_{i k}+\boldsymbol{V}_{i k}
$$

Then the estimation of the local filter in the two subsystems is $\hat{X}_{1}$ and $\hat{\boldsymbol{X}}_{2}$. The corresponding estimation variance matrix is $\boldsymbol{P}_{1}$, and $\boldsymbol{P}_{2}$. And the covariance matrix of noise is $\boldsymbol{Q}_{1}$ and $\boldsymbol{Q}_{2}$. The time update value of federated filter is $\hat{\boldsymbol{X}}_{m}$. The estimated error variance matrix is $\boldsymbol{P}_{m}$. The covariance matrix is $\boldsymbol{Q}_{m}$. The optimal global estimation of the system $\hat{\boldsymbol{X}}_{g}$ and $\boldsymbol{P}_{g}$ can be gained from (77)-(79).

$$
\begin{gathered}
\boldsymbol{P}_{g}^{-1} \hat{\boldsymbol{X}}_{g}=\boldsymbol{P}_{1}^{-1} \hat{\boldsymbol{X}}_{1}+\boldsymbol{P}_{2}^{-1} \hat{\boldsymbol{X}}_{2} \\
\boldsymbol{Q}_{g}^{-1}=\boldsymbol{Q}_{1}^{-1}+\boldsymbol{Q}_{2}^{-1} \\
\boldsymbol{P}_{g}^{-1}=\boldsymbol{P}_{1}^{-1}+\boldsymbol{P}_{2}^{-1}
\end{gathered}
$$

The feedback to each subsystem can be allocated according to the following rules:

$$
\begin{gathered}
\hat{\boldsymbol{X}}_{i}=\hat{\boldsymbol{X}}_{g} \\
\boldsymbol{Q}_{i}^{-1}=\xi_{i} \boldsymbol{Q}_{g}^{-1} \\
\boldsymbol{P}_{i}^{-1}=\xi_{i} \boldsymbol{P}_{g}^{-1} \\
\xi_{1}+\xi_{2}=1 \quad 0 \leq \xi_{i} \leq 1
\end{gathered}
$$

The federated filter consists of two parts: filtering \& estimation of local state, and information fusion of global state. As shown in Fig.6, the SINS/DVL and USB navigation subsystem outputs local estimation $\hat{X}_{1}$ and $\hat{X}_{2}$ of the system, the estimated variance matrix $\boldsymbol{P}_{1}$ and $\boldsymbol{P}_{2}$, and the noise variance matrix $\boldsymbol{Q}_{1}$ and $\boldsymbol{Q}_{2}$, respectively. Then we input the local estimation value into the main federated filter and fuse with the reference subsystem from the SINS. Finally, we get $\hat{\boldsymbol{X}}_{g}$ and $\boldsymbol{P}_{g}$, which is the best estimation of the global state. Finally, the federated filter output the global navigation and positioning result and feedback the SINS/DVL subsystem.

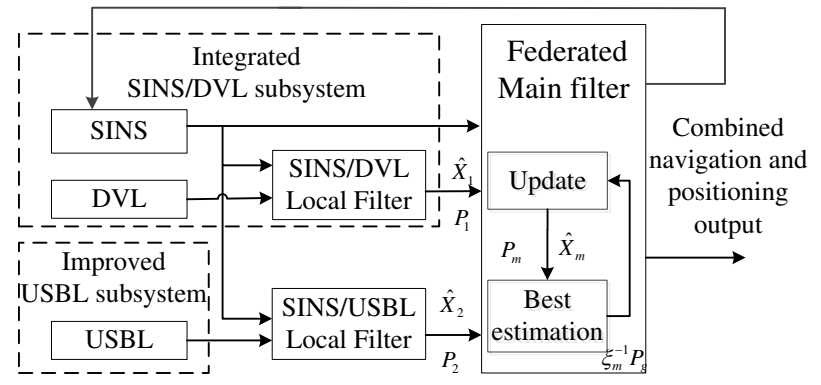

Fig. 6 The framework of the improved federated combined filtering based navigation and positioning system

The federated Kalman filter includes two stages: time update and information fusion. During the time update stage, the global status estimation $\hat{\boldsymbol{X}}_{m}$, estimated variance matrix of error $\boldsymbol{P}_{m}$, and covariance matrix $\boldsymbol{Q}_{m}$ will be updated. And during the information fusion stage, we combine the status estimation of global federated main filter and estimation of local state of each subsystem. Finally, the filter assigns information in a specific way, feedback the best estimation of global status and its variance matrix to each navigation and positioning system.

\section{PERFORMANCE EVALUATION}

In this section, we evaluate the performance of our proposed integrated navigation and positioning system based on federated Kalman filter. We first set up the evaluation environment. Then we measure the performance of the method, including the positioning accuracy, stability, and performance. We also make comparisons with other related method.

\section{A. Simulation Setup}

(1) Evaluation environment

We first set up a Matlab simulation environment. To validate and evaluate the accuracy and stability of the filtering procedure, we set up a motion model with time-varying motion states. We indicate the motion velocity and motion trace in Fig. 7 and Fig. 8. More specifically, the total time of motion is $2000 \mathrm{~s}$. An AUV first moves in the direction of the northeast. The speed in the north is $2.57 \mathrm{~m} / \mathrm{s}$, while the speed in the east is $0.27 \mathrm{~m} / \mathrm{s}$. After $1000 \mathrm{~s}$, the AUV changes the direction to the east in the sale of $3^{\circ} / \mathrm{s}$. After $600 \mathrm{~s}$, the AUV change the direction to the north at the scale of $3 \% / s$. it lasts 25 s. Finally, the UUV moves straightly. From 1000s to $2000 \mathrm{~s}$, the velocity is $2.57 \mathrm{~m} / \mathrm{s}$. We conduct simulations based on the motion model and the data. 


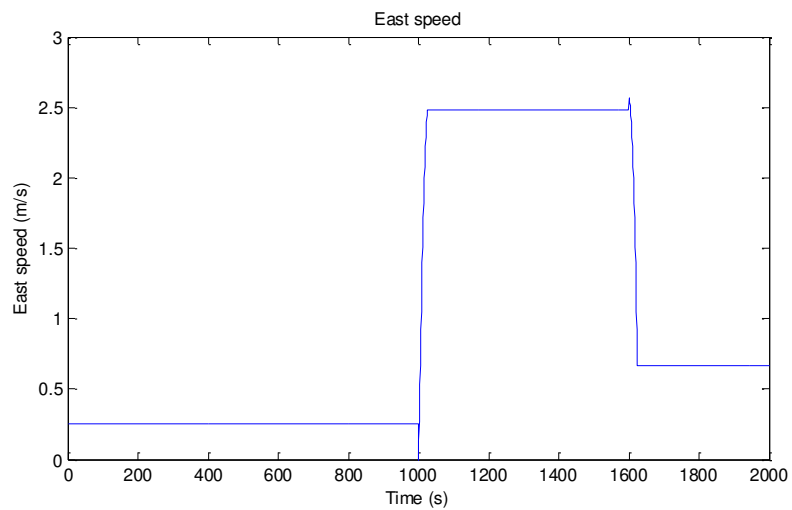

Fig. 7 The motion velocity of evaluation

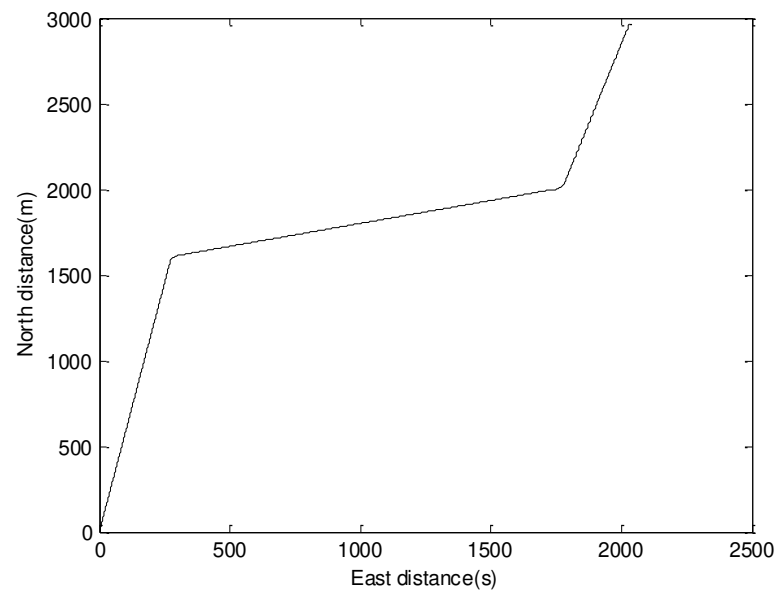

Fig. 8 The motion of the combined navigation and positioning system velocity of evaluation

\section{(2) Evaluation parameters}

We evaluate the positioning accuracy regarding the positioning error, the eastern velocity error and the mean square error. The lower the positioning error is, the higher the positioning accuracy is.

For positioning efficiency, we evaluate the computation time of navigation and positioning. Short processing time indicates high positioning efficiency.

(3) Reference Methods

We select the improved SINS/DVL positioning method [4] and the federated Kalman filter-based positioning [26] (note as classic FKF) as the reference methods. We call our proposed federated-filter-based positioning method as improved FKF.

\section{B. Positioning Accuracy evaluation}

We evaluate the position accuracy with the improved SINS/DVL positioning method, the classical federated filterbased positioning method.

(1) Compare with improved SINS/DVL

With the simulation environment and the motion model of UUV, we simulate the movement of UUV and perform the federated filter-based positioning. We evaluate the positioning accuracy of the positioning method and compare it with that of the improved SINS/DVL positioning method as shown in Fig. 9. 


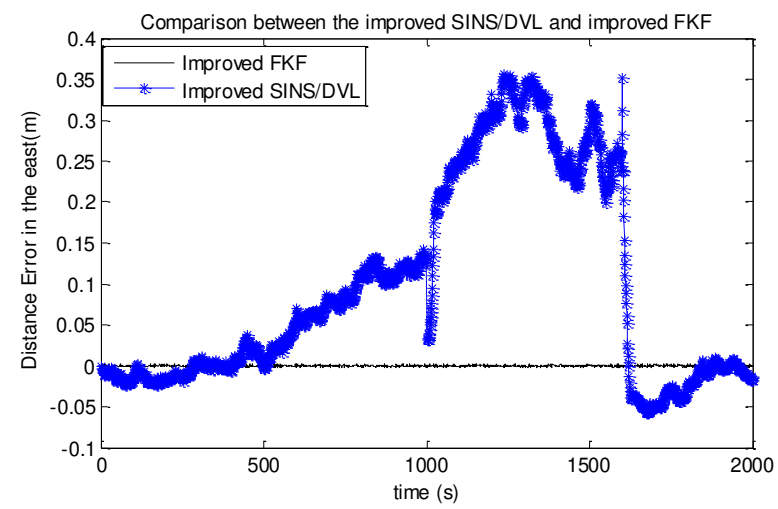

Fig. 9 The comparison between the improved SNIS/DVL and the improved FKF

From Fig. 9, we can conclude that the proposed federated filter-based positioning method gains higher accuracy. More specifically, the average distance error of the improved SINS/DVL is $0.20 \mathrm{~m}$ in the direction of the east, while that of our proposed SINS/DVL/USBL federated filter is $0.02 \mathrm{~m}$. Positioning accuracy is improved almost ten times. We also compare the positioning error of the two positioning methods. The average positioning error of the proposed SINS/DVL/USBL federated filter is less than $0.01 \mathrm{~m}$, while the average positioning error is $0.27 \mathrm{~m}$. Although it restrains the positioning error when the state changes rapidly at the time of $1000 \mathrm{~s}$, at the time of $1600 \mathrm{~s}$, it could not restrain the positioning error caused by the state change rapidly. For federated-filter based combined navigation and positioning

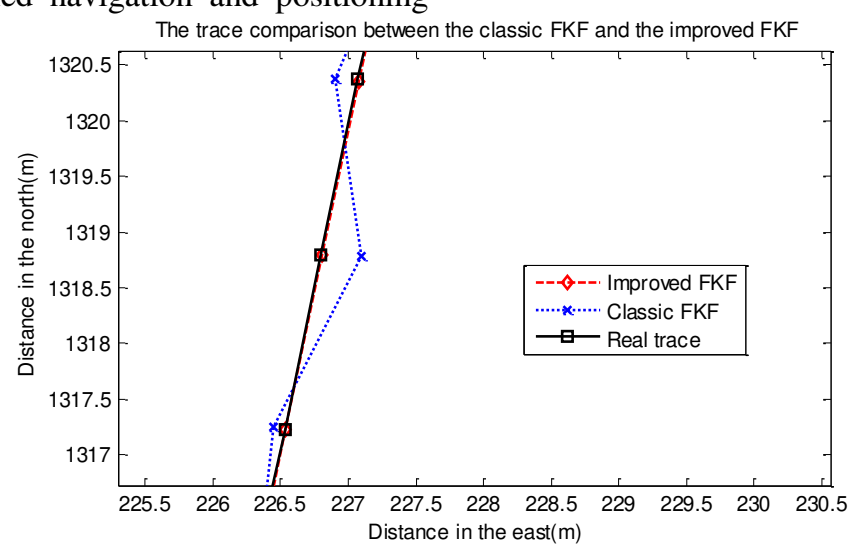

Fig. 10 The performance comparison of the two methods

Fig. 10 illustrates that the proposed improved federated filter-based navigation and positioning system gain lower positioning error than the classic federated filter. It can enhance the positioning accuracy on a scale of 4-5 times on average. That's because based on the classic federated filter, we conduct optimization on the navigation and positioning system. More specifically, we utilize the filtering gain compensation based adaptive filter, which can improve the positioning accuracy of the subsystem at a large scale. And the positioning accuracy of the whole navigation and positioning system is improved. system can strain the positioning error caused by the state change rapidly at the time of 1000 s and 1600 s. So the SINS/DVL/USBL combined navigation and positioning system can gain higher stability and reliability, especially when a complex environment.

(2) Compare with the federated-filter based positioning method

We also compare our proposed method with classic federated filter. With the same simulation setting, we compare the positioning error of the proposed integrated system with that of classic federated filter-based navigation and positioning system in Fig. 10.
We illustrate the east velocity error of the classical federated filter in Fig. 11. We can see that the improved federated filter-based navigation gains higher accuracy. More specifically, the east velocity error of the improved federated filter-based method is $1 / 3$ of that of classic federated filter. And the velocity error fluctuates is also tiny. That's because the subsystem in the improved federated filter adopts an improved adaptive filter making the system more stable and more accurate. 


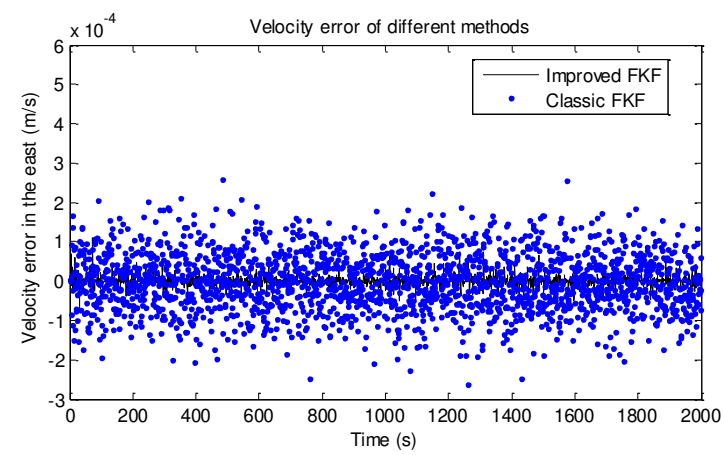

Fig. 11 The velocity error of the two methods

(3) MSE evaluation

We evaluate the mean square error (MSE) of the improved federated filter based positioning system. We also compare it with the improved SINS/DVL system [4] and FKF [26] in Table I.

TABLE I. THE MSE COMPARISON OF DIFFERENT METHODS

\begin{tabular}{|c|c|c|}
\hline Filter Method & East MSE $\left(\mathbf{m}^{\mathbf{2}}\right)$ & West MSE $\left(\mathbf{m}^{2}\right)$ \\
\hline Improved FKF & $2.016 \times 10^{-4}$ & $2.128 \times 10^{-4}$ \\
\hline FKF & $2.340 \times 10^{-3}$ & $2.547 \times 10^{-3}$ \\
\hline Improved SINS/DVL & $8.324 \times 10^{-3}$ & $7.927 \times 10^{-3}$ \\
\hline
\end{tabular}

From Table I, we can note that the two federated filterbased positioning systems gain lower MSE than the improved SINS/DVL system. And the improved federated filter has the lowest positioning error among these three methods. And the east MSE of the improved FKF is less than 2.016 $\times 10^{-4}$. Relative to FKF and improved SINS/DVL, the proposed improved FKF can reduce the east MSE by $91.38 \%$ and 97.58 , respectively. And it also lesson the west MSE by $91.65 \%$ and $97.32 \%$, respectively. That is mainly due to the validation of the multiple-source information fusion. The proposed navigation and positioning method has better positioning performance.

From the simulation above, the proposed improved federated filter-based navigation and positioning method gains high positioning accuracy than the two other positioning methods, especially in a complex motion environment.

\section{Positioning efficiency evaluation}

We evaluate the efficiency of the proposed improved federated filter-based navigation and positioning method in terms of the processing time. We also compare it with the other two methods as illustrated in Table II.
TABLE II. THE PROCESSING TIME COMPARISON OF DIFFERENT METHODS

\begin{tabular}{|c|c|}
\hline Filter Method & Processing time of 100 times \\
\hline Improved FKF & 2.2280 \\
\hline Classical FKF & 1.0921 \\
\hline Improved SINS/DVL & 0.3709 \\
\hline
\end{tabular}

From Table II, we can see that the processing time of the proposed improved federated method is the highest of the three methods. That means that the proposed method has the lowest efficiency. We also conclude that the federated filter method includes the proposed method and the classical method have lower efficiency than the improved SINS/DVL method. That is because the federated filter is the fusion of multiple positioning subsystem. And the processing time of the proposed method is double that of classic federated filter method. That's because the proposed method adopts a more complex filter algorithm, which affects the processing efficiency. However, considering the accuracy and stability improvement of the proposed federated filter based system comprehensively, the loss of efficiency is accepted.

\section{CONCLUSION}

To improve the accuracy and stability of AUV, we presented an improved federated filter-based navigation and positioning system in this paper. In the system, we first utilized filtering gain compensation based SINS/DVL subsystem to improve the positioning accuracy. We also explore the phase difference filtering based USBL subsystem. Then with the federated filter, we integrate the SINS/DVL/USBL positioning subsystem and perform multiple sources information fusion to gain higher accuracy and more stability navigation and positioning. The simulation result illustrated that the presented federated filter-based navigation and positioning system could achieve higher performance.

\section{COMPETING INTERESTS}


The authors declare that there is no competing interests.

\section{AVAILABILITY OF DATA AND MATERIALS}

We did not use any external data. The motion model parameters we used are clearly listed in the manuscript.

\section{AUTHORS' CONTRIBUTIONS}

All members of our team have different contributions to the research work. More specifically, Qinghua Luo and Xiaozhen and Chenxu Wang designed and performed the experiments, Zhiquan Zhou and Chenxu Wang supervised the work and analyzed the experimental results. Qinghua Luo, Xiaozhen Yan, and Yang Shao drafted the manuscript. Jianfeng Li setup the simulation environment, Cong $\mathrm{Hu}$ reviewed and polished the manuscript. Chuntao Wang and Jinfeng Ding provided helpful suggestions and revised the manuscript.

\section{ACKNOWLEDGMENT}

We sincerely thank the college of Information Science and Engineering of Harbin Institute of Technology, Weihai for providing research environment. We also thank Yipeng Yang and Chao Liu for their help.

\section{FUNDING}

This work is partly supported by the National Natural Science Foundation of China (Grant Number: 61671174 and 51909039), the Major Scientific and technological innovation project of Shandong Province of China(Grant Number:2020CXGC010705), Chinese Postdoctoral Science Foundation (Grant Number:2020M672123), and Post-doc Creative Funding in Shangdong Province (Grant Number:244312), Weihai Research Program of Science and Technology, The key lab of Weihai, the engineering research centre of Shandong province, The joint innovation centre of Shandong province, The Guangxi Key Laboratory of Automatic Detecting Technology and Instruments (Grant Number:YQ18206, YQ15203).

\section{CORRESPONDING AUTHOR}

Qinghua Luo

\section{ABBREVIATIONS}

\section{AUV Autonomous Underwater Vehicle \\ SINS Strap-down Inertial Navigation System \\ DVL Doppler velocity log \\ USBL ultra-short baseline \\ IoT Internet of Things \\ FKF Federated Kalman Filter \\ FUKF Federated Unscented Kalman Filter \\ RSS Received Signal Strength}

\section{REFERENCES}

[1] Liu S, Zhang T, Zhang J, Zhu Y (2021) A new Coupled Method of SINS/DVL Integrated Navigation Based on Improved Dual Adaptive Factors. IEEE Transactions on Instrumentation and Measurement 70:210-221. https://doi.org/ 10.1109/TCC.2015.2481401
[2] Luo Q, Yan X, Zhou Z, Wang C, Hu C (2021) An Integrated Navigation and Localization System. In: Proceedings of the IEEE international Conference on Smart Internet of Things (SmartIoT), Virtual Conference, 13-15 August 2021

[3] Guo S, He B, Feng C, Liu H, Yin F, Zhang X, Mu X, Li T, Yan T (2019) Fault Tolerant Multi-Sensor Federated Filter for AUV Integrated Navigation. IEEE Underwater Technology. pp 1-4. https://doi.org/10.1109/UT.2019.8734279

[4] Yan X, Luo Q, Yang Y, Liu S, Li H, Hu C (2019) ITL-MEPOSA: An Improved Trilateration Localization with Minimum Uncertainty Propagation and Optimized Selection of Anchor nodes for wireless sensor networks. IEEE Access 7(1):53136-53146. https://doi.org/10.1109/ACCESS.2019.2911032

[5] Ramezani H, Fazel F, Stojanovic M, Leus G (2015) Collision Tolerant and Collision Free Packet Scheduling for Underwater Acoustic Localization. IEEE Transactions on Wireless Communications 14(5):2584-2595. https://doi.org/10.1109/TWC.2015.2389220

[6] Luo Q, Ju C, Yan X, Hu C, Wang C, Ding J (2020) Accurate Underwater Localization Through Phase Difference. Paper presented at the 2020 IEEE International Conference on Smart Internet of Things (SmartIoT). pp 38-42. https://doi.org/10.1109/SmartIoT49966.2020.00015

[7] Morgado M, Oliveira P, Silvestre C, Vasconcelos J (2014) Embedded Vehicle Dynamics Aiding for USBL/INS Underwater Navigation System. IEEE Transactions on Control Systems Technology 22(1):322-330. https://doi.org/ 10.1109/TCST.2013.2245133

[8] Luo Q, Yan X, Ju C, Chen Y, Luo Z (2021) An Ultra-Short-Baseline Underwater Positioning System with Kalman Filtering, Sensors 21(1):143. https://doi.org/10.3390/s21010143

[9] Zhu Z, James Hu S, Li H (2016) Effect on Kalman based underwater tracking due to ocean current uncertainty. Paper presented at the IEEE/OES Autonomous Underwater Vehicles (AUV). pp 131-137. https://doi.org/10.1109/AUV.2016.7778660

[10] Qian Y, Chen Y, Cao X, Wu J, Sun J (2016) An underwater bearingonly multi-target tracking approach based on enhanced Kalman filter. Paper presented at the 2016 IEEE International Conference on Electronic Information and Communication Technology (ICEICT). pp 203-207. https://doi.org/ 10.1109/ICEICT.2016.7879684

[11] Xu J, Xiong Z, Liu J, Wang R (2019) A dynamic vector-formed information sharing algorithm based on two-state chi square detection in an adaptive federated filter. The Journal of Navigation 72(1): 101120. https://doi.org/10.1017/S0373463318000565

[12] Yang Y, Liu X, Zhang W, Liu X, Guo Y (2020) A nonlinear double model for multisensor-integrated navigation using the federated EKF algorithm for small UAVs. Sensors 20(10): 2974. https://doi.org/10.3390/s20102974

[13] Xiong H, Mai Z, Tang J, He F (2019) Robust GPS/INS/DVL navigation and positioning method using adaptive federated strong tracking filter based on weighted least square principle. IEEE Access 7: 26168-26178. https://doi.org/10.1109/ACCESS.2019.2897222

[14] Bao J, Li D, Qiao X, Rauschenbach T (2020) Integrated navigation for autonomous underwater vehicles in aquaculture: A review. Information Processing in Agriculture 7(1): 139-151. https://doi.org/10.1016/j.inpa.2019.04.003

[15] He C, Tang C, Yu C (2020) A federated derivative cubature Kalman filter for IMU-UWB indoor positioning. Sensors 20(12): 3514. https://doi.org/10.3390/s20123514

[16] Lei G, Fang Z, Luo B, Qi P (2018) A New Adaptive Federated Kalman Filter for the Multi-sensor Integrated Navigation System of MAVs. Paper presented at the 2018 13th World Congress on Intelligent Control and Automation (WCICA). pp 1804-1809. https://doi.org/10.1109/WCICA.2018.8630355

[17] Tang L, Tang X, Chen H, Liu X (2018) An Adaptive Federated Filter in Multi-source Fusion Information Navigation System. IOP Conference Series Materials Science and Engineering 392(6):062195. https://doi.org/10.1088/1757-899X/392/6/062195 
[18] Gong X, Zhang J (2016) An innovative transfer alignment method based on federated filter for airborne distributed POS. Measurement 86(1):165-181. https://doi.org/10.1016/j.measurement.2016.02.016

[19] Ma X, Zhang T, Liu X (2018) Application of Adaptive Federated Filter Based on Innovation Covariance in Underwater Integrated Navigation System. Paper presented at the 2018 IEEE International Conference on Manipulation, Manufacturing and Measurement on the Nanoscale (3M-NANO). pp 209-213. https://doi.org/10.1109/3MNANO.2018.8552184

[20] Xu J, Xiong Z, Liu J (2019) A Dynamic Vector-Formed Information Sharing Algorithm Based on Two-State Chi Square Detection in an Adaptive Federated Filter. Journal of Navigation 72(1):101-120. https://doi.org/10.1017/S0373463318000565

[21] Wang Q, Cui X, Li Y, Ye F (2017) Performance Enhancement of a USV INS/CNS/DVL Integration Navigation System Based on an Adaptive Information Sharing Factor Federated Filter. Sensors 17(2):239. https://doi.org/10.3390/s17020239

[22] Xu Q, Chang B, Li X, Liu X, Tian Y (2021) Vision-IMU Integrated Vehicle Pose Estimation based on Hybrid Multi-Feature Deep Neural Network and Federated Filter. Paper presented at the 2021 28th Saint Petersburg International Conference on Integrated Navigation
Systems (ICINS). pp

https://doi.org/10.23919/ICINS43216.2021.9470863

$1-5$.

[23] Li H, Ao L, Guo H, Yan X (2020) Indoor multi-sensor fusion positioning based on federated filtering. Measurement 154:107506. https://doi.org/10.1016/j.measurement.2020.107506

[24] Wang L, Wang S, Yang W (2021) Adaptive federated filter for multisensor nonlinear system with cross-correlated noises. Plos one 16(2):e0246680. https://doi.org/10.1371/journal.pone.0246680

[25] Yue Z, Lian B, Tang C, Tong K (2020) A novel adaptive federated filter for GNSS/INS/VO integrated navigation system. Measurement Science and Technology 31(8): 085102. https://doi.org/10.1088/1361$6501 / \mathrm{ab} 78 \mathrm{c} 2$

[26] Ayabakan T, Kerestecioğlu F (2021) RSSI-Based Indoor Positioning via Adaptive Federated Kalman Filter. IEEE Sensors Journal. pp 1-1. https://doi.org/10.1109/JSEN.2021.3097249

[27] Mikhail A., Leon H. D (2011) A design method and algorithm for USBL systems with skew three-element arrays, International Journal of Circuits, Systems and Signal Processing, 5(4): 382-390, May 2011. 